\title{
The Cognitive Load of Narrative Lies
}

Lisa Bird $^{1}$, Robert Cockerell ${ }^{2}$, Matthew Gretton ${ }^{1} \&$ Andrew Heathcote ${ }^{1}$

${ }^{1}$ Division of Psychology, University of Tasmania

${ }^{2}$ KordaMentha Forensic

1.a.bird@utas.edu.au

rcockerell@kordamentha.com

andrew.heathcote@utas.edu.au 
Lying is assumed to increase cognitive load, and it has been shown to slow response times to simple questions. We employed a dual-task methodology, the Detection Response Task (DRT), to assess cognitive load in telling narrative lies in a live, open-question interview. The DRT requires participants to press a button in response to a tactile stimulus every 3-5 seconds while simultaneously performing a primary task; in this case recounting either truthful or deceitful narratives. We found weak support for slowing in the time to initiate a narrative response when lying. In contrast, we found strong support for an increase in cognitive load when producing a narrative lie, as measured by both slowed DRT responses and increased response omissions, although this effect decreased with time on task. We advocate dual-task methodologies such as the DRT for increasing understanding of the assumptions made by theories of deception, and for refining lie-detection techniques.

Keywords: deception, cognitive load, Detection Response Task 
People engage in deceptive behaviours on a daily basis, from inconsequential white lies to high-stakes acts of dishonesty (DePaulo, Kashy, Kirkendol, Wyer, \& Epstein, 1996). Typically, studies of lying have focused on their detection; here we have a more theoretical focus, on the relationship between deception and cognitive load. Deception is thought to increase cognitive load because it requires hiding emotional responses (Ekman \& Frisen, 1969), formulating lies that are both internally consistent and consistent with what the recipient already knows (Zuckerman, DePaulo \& Rosenthal, 1981), watching for signs of suspicion in the recipient (Buller \& Burgoon, 1996), and in general engaging in impression management (DePaulo et al., 2003). Lying is also thought to require cognitive control, because it is subject to Stroop-like interference from a prepotent honest response (Pennebaker \& Chew, 1985). Consistent with these effects, subjective reports indicate lying is harder than telling the truth (Caso, Gnisci, Vrij, \& Mann, 2005).

Walczyk, Harris, Duck and Mulay’s (2014) Activation-Decision-Construction-Action theory (ADCAT) provides a detailed characterization of the cognitive processes involved in lying that implicates a number of loci for increased cognitive load. In ADCAT, the activation phase involves retrieval of the truth from long term memory. The decision involves central executive functions weighing up the consequences through a cost-benefit and likelihood analysis. During construction a plausible narrative is produced while conflicting details are inhibited. The action phase involves delivering the lie while monitoring the receiver for signs of disbelief and suppressing interference from the truth. These phases occur simultaneously, and all increase the time required to initiate a response.

Suchotzki et al. (2017) found response time (RT) to be a reliable measure of the increased cognitive load associated with deception in a meta-analysis of 73 studies, with a large effect size $(d=1.26)$. However, RT has traditionally only been tested with simple binary responses in tasks such as the Concealed Information Test (Seymour, Seifert, Shafto, 
\& Mosmann, 2000), and the Sheffield Lie Test (Spence et al., 2001), or occasionally with a mixture of binary and one- or two-word responses, such as in the Time Restricted Integrity Confirmation test (Walczyk et al. 2005). These tasks involve responding to an audio recording via a computer, but deception often occurs within a social context where it is blended with truth in the production of narratives (McCornack, Morrison, Paik, Wisner \& Zhu, 2014).

Here we examine RT in the production of narrative lies. Our participants viewed short films and recounted the content to an interviewer (in either a 'lie' or 'truth' condition). A cue presented before each video indicated if their recounting should be truthful or untruthful. If narrative lie responses require additional cognitive load, verbal RT (i.e., the delay between the end of the interviewer's question and the onset of the participant's response) should be longer in the lie than truth condition. This assertion underpins our first hypothesis preregistered on the Open Science Framework (https://osf.io/jt5pg/) ${ }^{1}$. We also employed a live interview rather than computer-presented questions in order to induce the cognitive load associated with impression management. We also told the participants that they would have to recall some details of their answers at the end in order to capture the extra cognitive load occurring in real-world situations; lies must be sufficiently memorable so that reporting remains consistent over repeated interrogations.

We took advantage of the temporally extended nature of our experiment to not only measure cognitive workload during the action stage (i.e., when producing a narrative) but also, for the first time to or knowledge, during the construction stage (i.e., when viewing the video). To do so we use a dual-task methodology, the Detection Response Task (DRT),

\footnotetext{
${ }^{1}$ In addition, we pre-registered additional hypotheses that have not yet been tested. The project for this research can be found at https://osf.io/jt5pg/. Data files for DRT and Vocal $\mathrm{RT}$, an RStudio project to reproduce all analyses and figures, and, movie URLs, are publicly available through the above link.
} 
which requires participants to press a button in response to an easily-perceived tactile stimulus that occurs every 3-5 seconds. The DRT has been adopted by the International Standards Organization as a measure of distracted driving (ISO 2016). Central to dual task methodologies is the notion that attentional resources are limited. If the primary task - in this case, lying - requires more attentional resources than are available, then performance on the secondary task - in this case the DRT - is hindered by having fewer resources to call on. The performance in the secondary task can then be used to determine the level of resources the primary task requires.

Slowed DRT responses and response omissions have been found to provide sensitive measures of the cognitive load associated with a range of cognitive processes that are likely to be involved in narrative deception, including decision making, sustaining information in working memory, overcoming habitual actions, and holding a conversation (Strayer et al., 2015; Tillman, Strayer, Eidels, \& Heathcote, 2017). Based on the assumption that constructing and recounting lies increases cognitive load, our second and third pre-registered hypotheses are that DRT RT and omission rates will be higher in the lie than truth condition. Our design enabled us to collect an average of over 800 DRT trials per person, greater than the minimum of 20 verbal RTs recommended by Suchotzki et al. (2017) who note that previous deception RT studies have been limited by very few RT measurements.

\section{Method}

\section{Participants}

Sample size was determined by specifying a fixed period of time for data collection (ending the second week of August with 44 participants). Participants were first year Psychology students (29 females) aged 18-66 $(M=27.1$ years, $S D=10.6)$ who were given course credit for participation. 


\section{Materials}

The DRT device conformed to ISO standard ISO DIS 17488 (ISO, 2016), using a tactile stimulus delivered by a $10 \mathrm{~mm}$ diameter 5 volt vibrating motor running at $11000 \mathrm{RPM}$ and housed in a 3D-printed casing $(14 \mathrm{~mm} \times 7 \mathrm{~mm})$. The housing was attached to the participant's clavicle with tape and vibrated at random intervals ranging from 3-5 seconds with uniform probability. Participants responded to the vibrations with a button (a $6 \mathrm{~mm}$ metal tactile ball) housed in a $16 \mathrm{~mm}$ square $3 \mathrm{D}$ printed housing which was strapped to the index finger of their dominant hand with a Velcro strap. The stimulus and response devices were connected to an Arduino Nano 3.0 compatible microcontroller running custom software which interfaced via serial-over-USB to the experiment application. Timing was millisecond accurate.

The Arduino-based software started and stopped the DRT protocol and reported events and timing information back to the PC experiment software. The software played, in random order, 26, one-minute long YouTube videos that were submitted as entries to 60 second film competitions (URLs available at https://osf.io/jt5pg/). All videos had a coherent story and were screened for explicit content. The experiment software was developed as a C\#/WPF .NET application and ran on a Windows 10 PC with a 1920x1080 24" monitor. The movies ran full-screen.

The DRT RTs were time-locked to the trials by referencing the computer clock readings for each DRT response saved in the DRT data file. Verbal RTs were coded using sound files in Adobe Audition (Adobe Systems 2018, version 11.1.1.3). By examining the waveforms for each trial, verbal RTs were calculated by manually marking the gap between speech at the end of the interviewer's question and the beginning of the participants' answer. 


\section{Procedure}

Participants were tested individually in a sound-attenuated room with a monitor and webcam. After having the task explained to them they read an information sheet, filled out a consent form, and were then fitted with headphones and the DRT. They were told that the content of the videos was unknown to the interviewer in order to encourage natural lies where the target does not necessarily know they are being lied to. Participants were instructed that they could tell lies either by producing a narrative that was completely unrelated to the video or take the main themes of the video and change as many details as possible. They were instructed that their lies needed to be convincing and plausible, and memorable as they would be asked to recall some responses after the task. They were told their responses would be checked after the task to determine if they were lying or not.

The interviewer sat in front of a webcam enabled computer in an adjacent room and interacted with the participant through Skype (Skype for Windows 10, Version 8.18.0.6). The interviewer could not see the participant's screen but throughout the experiment the participant and interviewer's faces were visible to each other.

The participant's computer presented each video and the DRT ran continuously throughout the experiment. Before each of the videos began a cue appeared on the participant's screen instructing them to either lie or tell the truth (e.g., "After the following movie, please tell the truth when you reply to the questions."). At the end of each movie a cue appeared ("Now signal to the interviewer that the movie has finished") with either Truth or Lie in bold at screen centre.

Participants did one practice trial each of the lying and truth conditions before the main task began. After each practice trial they were given verbal feedback such as "that level of detail is fine", or, if the response was only one sentence, to "remember to provide as much detail as you can”. There were no further prompts throughout the experiment. Once any 
participant questions were clarified they were instructed to follow the prompt on the screen ("Hold down Ctrl, left arrow and then the space bar to run the next video", which aimed to prevent them skipping ahead) to start the experimental trials. After the one-minute video, the prompt to lie/tell the truth returned and remained on the participant's screen. The interviewer then asked: "Please describe as quickly as possible in as much detail as possible, what occurred in the video". Once the answer was completed the interviewer asked: "As quickly as possible in as much detail describe the main character". When the response was brief the interviewer used a follow up question, such as "Can you describe one of the characters?" or, if they had described the characters in detail in the first response, "Can you describe what they were wearing?". The interviewer then asked the participants to follow the screen prompts for the next video.

The main experiment consisted of thirteen true and thirteen lie trials that were randomly ordered, except that after two consecutive lie or truth trials the software always switched to the alternative. A rest break occurred after trial fourteen. At the end of the experiment participants were asked to recount their answer to the trial that occurred before the break and the final trial. The total length of the experiment averaged 75 minutes.

\section{Results}

The responses were informally scrutinised by the trained interviewer throughout the task. Participants gave a variety of responses that contradicted the content of the videos and alternated between strategies of simply changing character genders, to constructing wholly new narratives. One participant consistently failed to provide deceptive narratives and so their data was excluded from further analysis.

Following the ISO standard (ISO, 2016), all DRT RTs faster than 0.1 secs (1.2\% of all responses) and slower than $2.5 \operatorname{secs}(1.2 \%$ of all responses) were considered anticipations or omissions and excluded, leaving an average of 822 valid DRT trials per participant. All 
DRT trials during the practice trials and session break were removed, as were those immediately before the onset of each movie (as participants had to hold down several buttons, which disabled their ability to respond to the DRT).

No censoring was performed on the verbal RT data as participants could prepare their answer while listening to the question and anticipate when it finished, so very fast responses are plausible. Similarly, slow responses are plausible, and they were not overly influential because the analysis was performed on a logarithmic scale where verbal RT distribution was approximately normal, with the same being true for DRT RT. Most participants had the maximum number of valid verbal RTs, 52 (two per trial), with one having 45 and another 50 .

For all analyses responses were divided into blocks before and after the break in order to test for time-on-task effects due to factors like practice and fatigue. Inference was carried out using linear mixed-effect models with the R package lme4 (Bates, Maechler, Bolker, \& Walker, 2014). RT analysis used a general linear model assuming Gaussian error for logarithmically transformed RT data, and DRT omissions analysis used a generalized linear mixed model assuming a binomial error model and a probit link function. In all analyses the 43 participants and 26 movies were included as additive random effects and block and veracity (truth vs. lie) were entered as fixed effects. For some DRT analyses a "phase" factor, (construction while watching the movie vs. action while providing a narrative response) was also a fixed factor. Fixed effects were assessed using a Type II Wald chi-square test as implemented by the car package (Fox \& Weisberg, 2011).

Bayesian analyses were carried out with the default prior method of Rouder, Morey, Speckman, and Province (2012) using the BayesFactor R package (Morey \& Rouder, 2018). For RT the same type of general linear model was used as for the frequentist analysis. As generalized linear models are not available in BayesFactor, we calculated omission probabilities for each trial using Snodgrass and Corwin's (1998) recommended edge 
correction, probit transformed them, and applied a general linear model analysis to these values. We report $95 \%$ confidence intervals in square brackets.

\section{DRT Performance}

RTs were significantly slower when lying $(M=440,[438-443])$ than when telling the truth $(M=447,[445-449]) \chi^{2}(1)=31.39, p<.001$ indicating higher load in the lie condition than the truth condition. RTs were also significantly slower in the narrative phase $(M=528$, [520-535] than in the construction phase $\left(M=387\right.$, [382-393], $\chi^{2}(1)=4441.18, p<.001$, indicating much higher load for reporting than constructing narratives.

Figure 1 shows evidence of pervasive fatigue effects, with RT slowing and omissions increasing from the first $(M=437,[433-442])$ to second half $(M=452,[447-456]$ of the experiment, as indicated by significant block main effects for both RT (Construction: $\chi^{2}(1)=$ 42.2, $p$ <.001; Narrative: $\chi^{2}(1)=7.4, p=.006$ ) and omissions (Construction: $\chi^{2}(1)=35.8, p$ $<.001$; Narrative: $\left.\chi^{2}(1)=123, p<.001\right)$. The block effect interacted with veracity in the narrative condition for both $\mathrm{RT}, \chi^{2}(1)=11.2, p<.001$ and omissions, $\chi^{2}(1)=5.7, p=.017$, but analogous interactions for the construction phase did not reach significance, $\chi^{2}(1)=0.9, p$ $=.33$, and $\chi^{2}(1)=1.7, p=.19$, respectively. In light of these findings we tested our hypotheses separately for each block.

In Block $1 \mathrm{RT}$ in the lie condition was significantly slower than for the truth condition both in the construction, $\chi^{2}(1)=6.3, p=.012$, and narrative, $\chi^{2}(1)=38.4, p<.001$, phases. In contrast, neither effect was significant in Block $2, \chi^{2}(1)=0.2, p=.64$ and $\chi^{2}(1)=1.55, p$ $=.21$, respectively. During the construction phase veracity did not have a significant effect on DRT omissions in either Block $1, \chi^{2}(1)=0.06, p=.8$, or Block $2, \chi^{2}(1)=2.05, p=.15$. In the narrative phase, however, omissions were significantly more common in the lie than truth condition, both in Block 1, $\chi^{2}(1)=27.8, p<.001$, and Block 2, $\chi^{2}(1)=4.6, p=.03$. 
Bayesian tests provided strong evidence not only of some differences, but also of equivalence. Block 1 differences for the narrative phase were supported by large Bayes Factors $(\mathrm{BFs}), \mathrm{BF}=3 \times 10^{6}$ for $\mathrm{RT}$, and $\mathrm{BF}=1.1 \times 10^{5}$ for omissions. The weaker Block 1 effect for RT in the construction phase was equivocal in the Bayesian analysis, $\mathrm{BF}=0.48$, whereas the analogous omission effect was associated with clear support for the null, BF = $0.091(1 / \mathrm{BF}=11)$. For RT in Block 2 there was strong support for a null effect in the construction phase, $\mathrm{BF}=.028(1 / \mathrm{BF}=35)$, and the narrative phase, $\mathrm{BF}=0.056(1 / \mathrm{BF}=$ 17.7). For omissions in Block 2 there was positive evidence for the null in the construction phase, $\mathrm{BF}=0.206(1 / \mathrm{BF}=4.86)$, and positive evidence for more lie than truth omissions in the narrative phase, $\mathrm{BF}=5.75$.

\section{Verbal RT}

One participant answered the questions before the interviewer finished speaking, giving no discernible break in speech pattern and their data was excluded. The audio recording failed for another, leaving data from 41 participants. As shown in Figure 2, participants were slower to respond when lying $(M=516,[455-584])$ than telling the truth $(M$ $=483,[424-550])$, in both the first and second halves of the experiment $\chi^{2}(1)=7.60, p$ $=.006$. Neither the block effect nor the interaction of block and veracity were significant. The same model performed the best in the Bayesian analysis, but its advantage over an interceptonly model was equivocal, $\mathrm{BF}=1.29$. Frequentist simple effect tests found a significant effect of veracity in Block 1, $\chi^{2}(1)=5.6 p=.018$, but not Block 2, $\chi^{2}(1)=2.34, p=.13$. However, the former effect was equivocal in a Bayesian analysis, $\mathrm{BF}=1.01$, and the latter effect provided positive evidence for the null, $\mathrm{BF}=0.2(1 / \mathrm{BF}=5)$. 


\section{Discussion}

We employed a widely used and validated dual-task methodology, the DRT (ISO 2016), to quantify the cognitive load caused by one type of deception that has not previously been much studied, narrative lies. For the first half of our experiment both frequentist and Bayesian analyses of DRT performance strongly supported the hypothesis that the production of narrative lies increases cognitive load, both in the form of slowed responses and response omissions. However, this effect was weakened in the second half of the experiment, with the elevation in omissions remaining significant and receiving positive but not strong support from the Bayesian analysis, whereas the Bayesian analysis strongly supported a null effect on RT. These results demonstrate that delivering narrative lies can require more effort than making an honest response, particularly when delivering lies with minimal rehearsal.

The frequentist analysis of DRT RT, but not omissions, supported a higher load for lie construction in the first half of the experiment, whereas in the Bayesian analysis the RT effect was equivocal, and the null was supported for omissions. For construction in the second half of the experiment there was no frequentist support in either measure for a veracity effect, and there was support for null effects in the Bayesian analysis. Overall, these results suggest that, at least in our paradigm, lie construction causes only a mild initial elevation in cognitive load, and that any effect that was present disappeared with time on task.

There are likely several factors involved in the diminished effect over the experiment. Firstly, rehearsal impacts the cognitive effort involved in lying (Walczyk et al., 2013). It is possible that increasing familiarity with the experiment allowed participants to take advantage of the construction phase to rehearse their lies. Second is the role of effort. Participants may have produced less complex lies as they became more familiar with the task. Reduction in complexity of lies over the task would also be supportive of the model of lying 
suggested by Information Manipulation Theory 2 (IMT2, McCornack, et al. 2014), that lies are only harder when they contain the portrayal of complex information, are based on the retrieval of decayed semantic memories, and when there has been no opportunity to rehearse the delivery.

Unfortunately, the nature of narrative lies makes measuring their complexity a challenge. According to IMT2, lies differ by their quality, quantity, manner and relevance and they are blended in truth (McCornack, et al. 2014). This is particularly true in narrative lies, which is a likely reason for the near absence of studies on them in the literature. Consistent with IMT2, participants gave a variety of lies, from as little as changing the gender of the main character to constructing wholly new narratives. Classifying these responses along a continuum from completely true to completely false would be a highly subjective endeavour. However, as a proxy for effort we analyzed overall time spent lying. On average, participants did not differ significantly between block 1 (505 ms) to block 2 (535 $\mathrm{ms}), p=.47$, and a Bayes factor of 0.2 indicated positive evidence for no difference. Even so, it remains possible that if we had required a homogenously high level of deception, rather than allowing participants to lie most comfortably for them, we may have obtained stronger effects.

Further, one aspect of constructing a plausible lie is not producing information the interviewer knows to be false. Our paradigm did not require this of participants, making lie construction easier than is usually the case. The clear lack of any construction effect during the second half of the experiment, and the reduced effect in the production phase, may be due to participants realising that they need not worry about the interviewer's evaluations.

Clearly, future research should address these limitations before a strong conclusion can be made that narrative lie construction produces little or no increase cognitive workload. Another interesting avenue for future research is to give participants a longer time to prepare 
their lies. This could potentially shift the balance of cognitive load onto the construction phase and away from the production phase.

A potential limitation of the DRT, and other dual-task methodologies, is that they may affect the primary task. However, the impact of the DRT on driving when it is the primary task have been shown to be minimal (Castro, Cooper \& Strayer, 2016). The same seems likely true of narrative production, which like driving, requires very different responses than the DRT. The DRT is also not suitable for the study of lies whose production is very brief, such as when giving one-word answers. The study of lying is challenging because of the great diversity of ways in which deception can occur, so it is likely that no one approach will be generally applicable. Fortunately, at least for complex and lengthy acts of deception - a characteristics that Walczyk, Roper, Seemann and Humphrey (2003) noted makes accounting for narrative lies a "formidable theoretical challenge" (p.757) - the DRT does seem well suited, and so can aid researcher in addressing the challenges presented by this diversity.

Our frequentist analysis showed an increase in verbal RT (i.e., the time to initiate a response in answer to a question) when lying in the first half of our experiment. However, this was not supported by a Bayesian analysis, and the effect disappeared in the second half. This finding stands in contrast to the strong effects reported by Suchotzki et al.'s (2017) meta-analysis of non-narrative lies, where there was an average effect of $0.115 \mathrm{sec}$, whereas we found an average effect of only $0.023 \mathrm{sec}$ in the first half of the experiment. Our paradigm afforded participants an opportunity for some preparation, whereas in the paradigms examined by Suchotzki et al. the instruction to lie was provided simultaneously with the question. The simple responses required in their paradigms mean the construction of a complex lie is not necessary, perhaps suggesting this greater slowing is associated with the decision to lie and, possibly, the need to supress the truth. In our paradigm both of these tasks 
can be accomplished before a response has to be initiated, and in many naturalistic contexts even more preparation time can be available.

A methodological limitation of verbal RT in the narrative context is that it is hard to collect many responses. Although we collected more than the minimum recommended by Suchotzki et al. (2017), this number was still relatively modest. The DRT afforded many more trials and hence far more precise individual measurement, recommending it as a better method to investigate cognitive load (see Smith \& Little, 2018, on the importance of precise individual measurement for reproducibility).

A further important advantage of dual-task methodologies like the DRT is that they provide a concrete grounding for the concept of cognitive load, which can otherwise be theoretically empty (Navon, 1984). This grounding may be particularly useful in assessing the influential Cognitive-Load Approach to lie detection, which attempts to make involuntary signs of lying more evident through manipulations aiming to increase cognitive load (e.g., recounting stories backward, engaging in unanticipated questions and tasks and forcing direct eye contact during interviews (see Vrij, Granhag, Mann, \& Leal, 2011). The DRT has the potential to provide a rigorous assessment of which methods of inducing cognitive load have the strongest effects. Although the voluntary nature of the DRT, like other RT-based approaches, likely makes it subject to counter measures, and so unsuitable for direct use in lie detection (Sip et al., 2013), we conclude that it does present a promising avenue toward refining both our theoretical understanding of deception and the techniques employed to make involuntary signs of lying more evident. 


\section{References}

Bates, D., Maechler, M., Bolker, B., \& Walker, S. (2014). lme4: Linear mixed-effects models using Eigen and S4. R package version 1.1-7. Retrieved from http://CRAN.Rproject.org/packagelme4

Buller, D. B., \& Burgoon, J. K. (1996). Interpersonal Deception Theory. Communication Theory, 6(3), 203-242. doi:10.1111/j.1468-2885.1996.tb00127.x

Caso, L., Gnisci, A., Vrij, A., \& Mann, S. (2005). Processes underlying deception: An empirical analysis of truth and lies when manipulating the stakes. Journal of Investigative Psychology and Offender Profiling, 2, 195-202. doi: http://dx.doi.org/10.1002/jip.32

Castro, S., Cooper, J., \& Strayer, D. (2016). Validating two assessment strategies for visual and cognitive load in a simulated driving task. In Proceedings of the Human Factors and Ergonomics Society Annual Meeting, 60, pp. 1899-1903, Sage, Los Angeles.

DePaulo, B. M., Kashy, D. A., Kirkendol, S. E., Wyer, M. M., \& Epstein, J. A. (1996). Lying in everyday life. Journal of Personality and Social Psychology, 70, 979-995. doi:

DePaulo, B. M., Lindsay, J. J., Malone, B. E., Muhlenbruck, L., Charlton, K., \& Cooper, H. (2003). Cues to deception. Psychological Bulletin, 129(1), 74-118. doi:10.1037/00332909.129.1.74

Ekman, P., \& Friesen, W. V. (1969). Nonverbal leakage and clues to deception. Psychiatry, 32, 88-106. doi: 10.1080/00332747.1969.11023575

Fox, J., \& Weisberg, S. (2011). An R Companion to Applied Regression (2nd ed.). Thousand Oaks, CA: Sage

ISO (International Organization for Standardization). (2016). Road Vehicles-Transport Information and Control Systems-Detection-Response Task (DRT) for Assessing Attentional Effects of Cognitive Load in Driving - 17488. ISO: Geneva, Switzerland.

McCornack, S. A., Morrison, K., Paik, J. E., Wisner, A. M., \& Zhu, X. (2014). Information Manipulation Theory 2. Journal of Language and Social Psychology, 33(4), 348-377. doi:10.1177/0261927x14534656

Morey, R. D. (2008). Confidence intervals from normalized data: A correction to Cousineau (2005). Tutorial in Quantitative Methods for Psychology, 4, 61-64.

Morey, R. D., \& Rouder, J. N. (2018). BayesFactor: Computation of Bayes Factors for Common Designs. R package version 0.9.12-4.2. https://CRAN.Rproject.org/package $=$ BayesFactor

Navon, D. (1984). Resources--a theoretical soup stone? Psychological Review, 91(2), 216234. http://doi.org/10.1037/0033-295x.91.2.216

Pennebaker, J. W., \& Chew, C. H. (1985). Behavioral inhibition and electrodermal activity during deception. Journal of Personality and Social Psychology, 49, 1427-1433. http://dx.doi.org/10.1037/0022- 3514.49.5.1427.

Rouder, J. N., Morey, R. D., Speckman, P. L., \& Province, J. M. (2012). Default Bayes factors for ANOVA designs. Journal of Mathematical Psychology, 56(5), 356-374. http://doi.org/10.1016/j.jmp.2012.08.001

Seymour, T. L., Seifert, C. M., Shafto, M. G., \& Mosmann, A. L. (2000). Using response time measures to assess "guilty knowledge". Journal of Applied Psychology, 85(1), 30-37. doi:10.1037/0021-9010.85.1.30

Sip, K. E., Carmel, D., Marchant, J. L., Li, J., Petrovic, P., Roepstorff, A., . . Frith, C. D. (2013). When Pinocchio's nose does not grow: Belief regarding lie-detectability modulates production of deception. Frontiers in Human Neuroscience, 7, 16. http://dx.doi.org/10.3389/fnhum.2013.00016 
Snodgrass, J. G., \& Corwin, J. (1988). Pragmatics of measuring recognition memory: applications to dementia and amnesia. Journal of Experimental Psychology: General, $117(1), 34$.

Spence, S. A., Farrow, T. F. D., Herford, A. E., Wilkinson, I. D., Zheng, Y., \& Woodruff, P. W. R. (2001). Behavioural and functional anatomical correlates of deception in humans. NeuroReport, 12, 2849-2853. http:// dx.doi.org/10.1097/00001756200109170-00019

Strayer, D. L., Turrill, J., Cooper, J. M., Coleman, J. R., Medeiros-Ward, N., \& Biondi, F. (2015). Assessing Cognitive Distraction in the Automobile. Human Factors: The Journal of the Human Factors and Ergonomics Society, 57(8), 1300-1324. doi:10.1177/0018720815575149

Suchotzki, K., Verschuere, B., Van Bockstaele, B., Ben-Shakhar, G., \& Crombez, G. (2017). Lying takes time: A meta-analysis on reaction time measures of deception. Psychological Bulletin, 143(4), 428-453. doi:10.1037/bul0000087

Tillman, G., Strayer, D., Eidels, A. et al. (2017). Modelling cognitive load effects of conversation between a passenger and driver. Attention, Perception, \& Psychophysics, 79(6), 1795-1803. doi:10.3758/s13414-017-1337-2

Verschuere, B., Crombez, G., De Clercq, A., \& Koster, E. (2004). Autonomic and behavioural responding to concealed information: differentiating orienting and defensive responses. Psychophysiology, 41(3), 461-466. doi:10.1111/j.14698986.00167.x

Vrij, A., Granhag, P. A., Mann, S., \& Leal, S. (2011). Outsmarting the Liars: Toward a Cognitive Lie Detection. Approach. Current Directions in Psychological Science, 20(1), 28-32. doi:10.1177/0963721410391245

Walczyk, J. J., Harris, L. L., Duck, T. K., \& Mulay, D. (2014). A social-cognitive framework for understanding serious lies: Activation-decision-construction-action theory. New Ideas in Psychology, 34, 22-36. doi:10.1016/j.newideapsych.2014.03.001

Walczyk, J. J., Roper, K. S., Seemann, E., \& Humphrey, A. M. (2003). Cognitive mechanisms underlying lying to questions: response time as a cue to deception. Applied Cognitive Psychology, 17(7), 755-774. doi:10.1002/acp.914

Walczyk, J. J., Schwartz, J. P., Clifton, R., Adams, B., Wei, M., \& Zha, P. (2005). Lying person to person about life events: A cognitive framework for lie detection. Personnel Psychology, 58(1), 141-170. doi:10.1111/j.1744-6570.2005.00484.x

Zuckerman, M., DePaulo, B. M., \& Rosenthal, R. (1981). Verbal and nonverbal communication of deception. In L. Berkowitz (Ed.), Advances in experimental social psychology (Vol. 14, pp. 1-59). New York: Academic Press. 

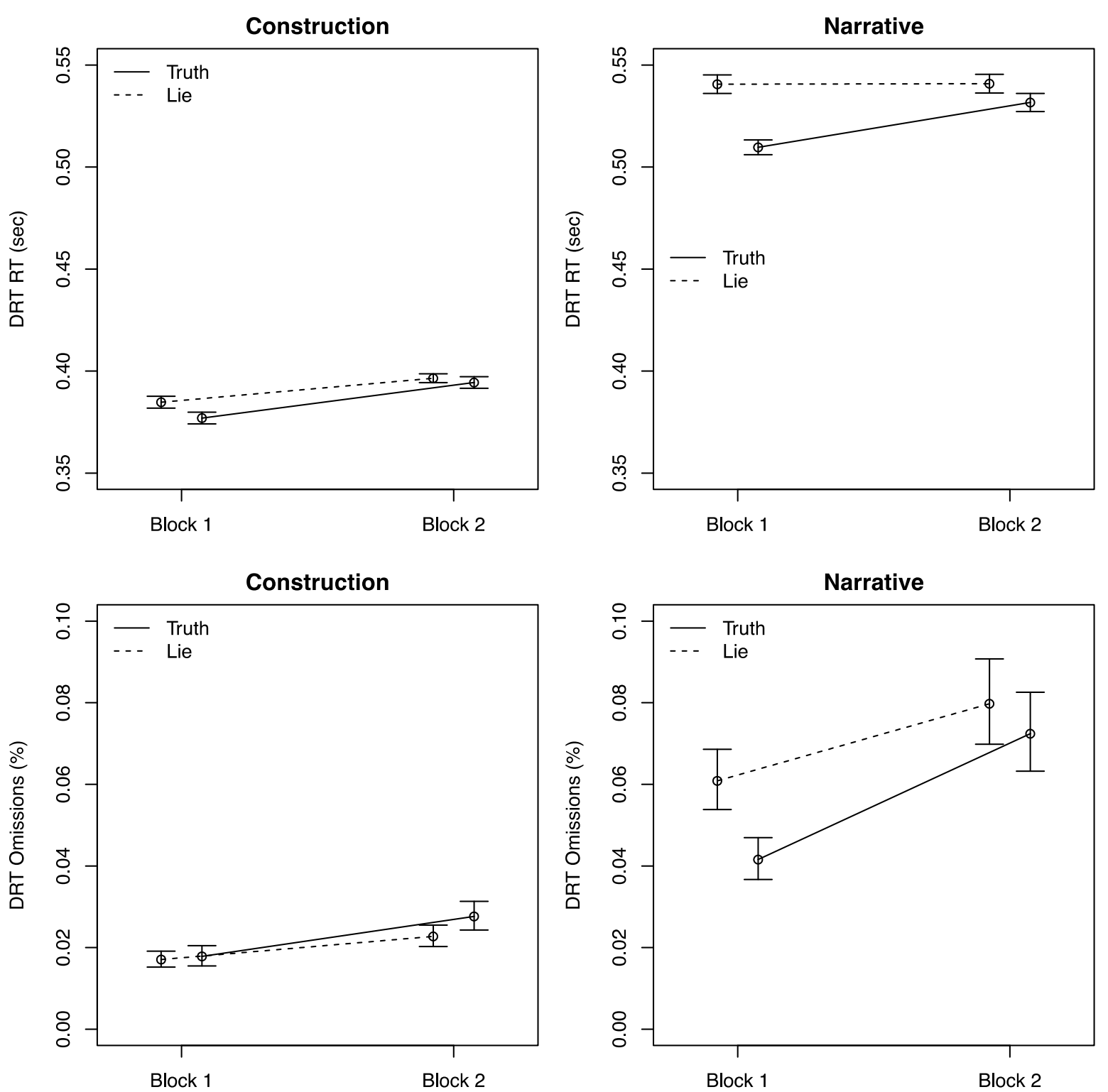

Figure 1. DRT mean RT (top row) and omission rate (bottom row) when watching the video (Construction, left column) and producing a response (Narrative, right column) for true and lie responses during the first (Block 1) and second (Block 2) half of the experiment. Means and within-subject standard errors (Morey, 2008) were calculated on the logarithmic scale for RT and on the probit for omission probability and the resulting points and intervals transformed back to the natural scale for graphing. 


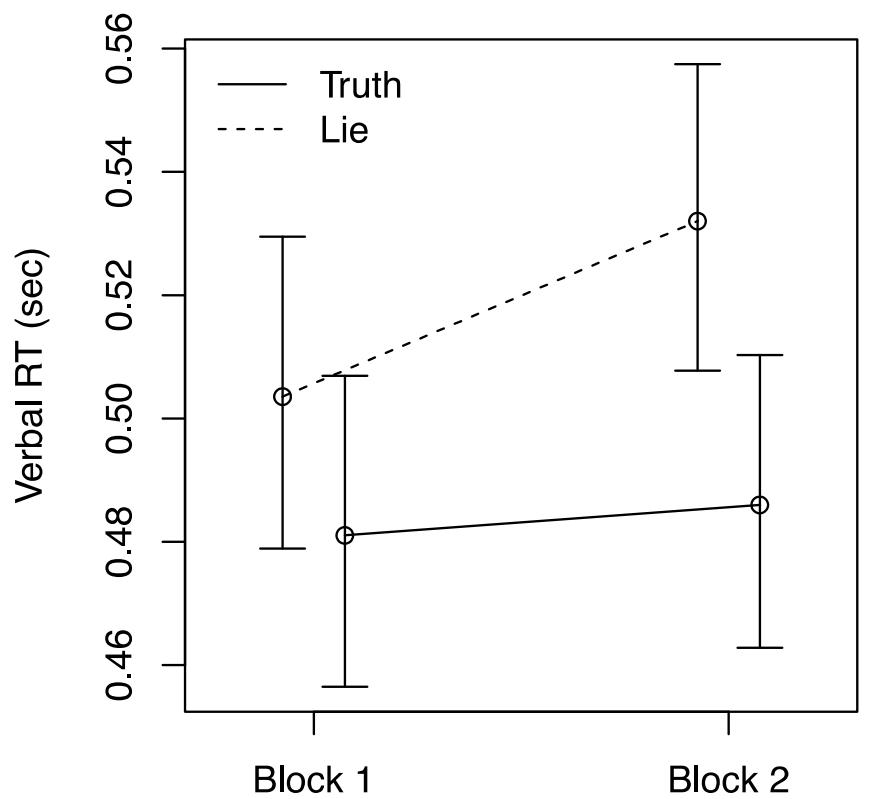

Figure 2. Mean time to answer questions for true and lie responses during the first (Block 1) and second (Block 2) half of the experiment. Means and within-subject standard errors (Morey, 2008) were calculated on the logarithmic scale for RT and the resulting points and intervals transformed back to the natural scale for graphing. 